\title{
ANÁLISIS CITOGENÉTICO EN POBLACIONES DE ALSTROEMERIA HOOKERI LODD. SSP. HOOKERI (ALSTROEMERIACEAE) EN LA REGIÓN DEL BÍO-BÍO, CHILE
}

\author{
CYTOGENETIC ANALYSIS IN POPULATIONS OF ALSTROEMERIA \\ HOOKERI LODD. SSP. HOOKERI (ALSTROEMERIACEAE) FROM BIO-BIO \\ REGION, CHILE
}

\author{
Daniel Cajas, Carlos Baeza, Eduardo Ruiz \& María Negritto \\ Departamento de Botánica, Facultad de Ciencias Naturales y Oceanográficas, \\ Universidad de Concepción, Casilla 160-C, Concepción, Chile. \\ cbaeza@udec.cl
}

\section{RESUMEN}

\begin{abstract}
Alstroemeria L. (Alstroemeriaceae) es un género de monocotiledóneas americano, con dos centros de distribución en el continente: Brasil y Chile. En Chile está representado por alrededor de 33 especies, la gran mayoría de ellas distribuidas en la zona central del país, área reconocida por su alto nivel de endemismo. El "complejo" Alstroemeria hookeri es endémico de Chile y se distribuye desde la IV Región de Coquimbo hasta la VIII Región del Bío-Bío. La subespecie hookeri está presente en la VII Región del Maule y en la VIII Región del Bío-Bío. En esta última existen dos centros de distribución de esta subespecie separados por la Cordillera de la Costa. Un centro se ubica en la zona costera de la región y el otro centro está ubicado en el interior, específicamente en la zona arenosa del valle central. El análisis citogenético de los cromosomas de las poblaciones costeras y del interior ha evidenciado una notable diferencia en el cariotipo, principalmente en el cromosoma 3. En las poblaciones costeras este cromosoma es telocéntrico mientras que en las poblaciones del interior es metacéntrico. También se observan diferencias en los cromosomas 4 y 8 , en las poblaciones costeras el cromosoma 4 es telocéntrico, y el 8 es submetacéntrico, en cambio en las poblaciones del interior tanto el cromosoma 4 como el 8 son subtelocéntricos. Al comparar índices de asimetría del cariotipo entre las poblaciones, tales como AsK\%, TF\% y Syi, se observan claras diferencias. El análisis fenético efectuado con datos citológicos también confirma la separación de las poblaciones costeras y del interior de Alstroemeria hookeri ssp. hookeri. Es probable que esté ocurriendo divergencia evolutiva entre las poblaciones costeras y del interior en esta subespecie y que la diferencia en la morfología del cromosoma 3 sea una evidencia morfológica de esta situación. Estudios preliminares de isoenzimas, RAPD, ITS y morfología corroboran esta proposición.
\end{abstract}

Palabras ClaVE: Alstroemeriaceae, “complejo Alstroemeria hookeri”, endemismo, citogenética.

\section{ABSTRACT}

Alstroemeria L. (Alstroemeriaceae) is an American genus of monocots, with two distribution centers: Brasil and Chile. In Chile is represented by about 33 species, the most of them in central Chile, an area renowned for its high level of endemism. The "complex" Alstroemeria hookeri is endemic to Chile and distributed from the IV Region of Coquimbo to VIII Region of Bío-Bío. The subspecies hookeri is present in the VII Region of Maule and VIII Region of Bío-Bío. In the VIII Region of Bío-Bío, there are two distribution centers of this subspecies separated by the Coastal Mountain. One center is located in the coastal range of the Region and the other center is located in the Central Valley, specifically on the sandy area of the Central Valley. The cytogenetic analysis of the chromosomes of coastal and eastern populations have shown a remarkable difference in the karyotype, principally in chromosome 3. In the Coastal range populations this chromosome is telocentric while in the Eastern populations is metacentric. Besides, there are differences in chromosomes 4 and 8 , in the Coastal ranges populations the chromosome 4 is telocentric and the chromosome 8 is submetacentric while in the Eastern populations both chromosomes are subtelocentric. When indexes of asymmetry of karyotypes are compared among populations (AsK\%, TF\% and Syi), there are clear differences between populations from both geographical range. The phenetic analysis using cytological data also confirms the separation of the coastal and inland populations within Alstroemeria hookeri ssp. hookeri. It is probably that evolutionary divergence between 
populations is occurring and the difference in the morphology of chromosome 3 is a morphological evidence for this situation. Preliminary studies of isoenzymes, RAPD, ITS and morphology support this hypothesis.

Keywords: Alstroemeriaceae, “Alstroemeria hookeri complex”, endemism, cytogenetic.

\section{INTRODUCCION}

Alstroemeria L. (Alstroemeriaceae) es un género exclusivamente sudamericano, que comprende alrededor de 50 especies, distribuidas desde Venezuela hasta Argentina y Chile, y que habitan desde el nivel del mar hasta los $4.500 \mathrm{~m}$ de altitud (Aagesen \& Sanso 2003, Ravenna 1988, Sanso 2002). El centro de diversidad del género se encuentra en Chile central (Bayer 1987), con una distribución satélite en el centro y este de Brasil (Aker \& Healy 1990). En Chile, el género está representado por 31-33 especies (Bayer 1987, Muñoz \& Moreira 2003) distribuidas desde el extremo norte hasta la Patagonia, siendo la zona central la que presenta la mayor concentración de especies (Muñoz \& Moreira 2003). Es uno de los géneros más diversificados y numerosos de la flora vascular chilena, lo que se traduce en una enorme y amplia variabilidad morfológica y florística, sobre todo en la coloración y ornamentación de sus flores, según queda de manifiesto en el trabajo de Muñoz y Moreira (2003).

Un aspecto importante de las especies de Alstroemeria en Chile es su distribución geográfica. De los 49 taxones que crecen en Chile, 42 se distribuyen en la zona central del país, entre los $28^{\circ}$ y $37^{\circ} \mathrm{S}$, sólo 3 especies se distribuyen hacia el norte de los $28^{\circ} \mathrm{S}$ y cuatro hacia el sur de los $37^{\circ} \mathrm{S}$. La gran diversidad de ambientes que ofrece esta región del país ha derivado en un alto nivel de endemismo (Arroyo 1995).

Dada la belleza de sus flores, las especies de Alstroemeria chilenas han adquirido relevancia mundial como plantas ornamentales de cultivo y de corte (Buitendijk \& Ramanna 1996, Buitendijk et al. 1997) y muchas de las especies han alcanzado un alto valor comercial(Stephens et al. 1993). El conocimiento cariológico de las especies nativas de Chile es, entonces fundamental para la obtención de nuevos genotipos con valor económico (forma, tamaño y color de las flores, así como la durabilidad y tamaño de las plantas). Los estudios cromosómicos en Alstroemeria comienzan con el trabajo de Strasburger (1882). Hasta el año 1989 el número de trabajos citológicos involucró a no más de 10 especies diferentes (Tsuchiya \& Hang 1989). En los últimos 20 años, este género ha sido estudiado citogenéticamente desde distintos puntos de vista, por ejemplo: localización física de secuencias de ADN repetitivas (De Jeu et al. 1997, Kamstra et al. 1997, Kuipers et al. 1997, 1998, 2002, Zhou et al. 2003, Baeza et al. 2007a), bandeo C (Buitendijk \& Ramanna 1996), mitosis y meiosis (Stephens et al. 1993), tamaño y variación del genoma (Buitendijk et al. 1998) y cariotipos (Sanso \& Hunziker 1998, Sanso 2002, Jara et al. 2004, Baeza et al. 2006, 2007b).

En Chile Alstroemeria hookeri es un complejo integrado por cuatro subespecies que ocupan un área de distribución que abarca desde la IV Región de Coquimbo a la VIII Región del Bío-Bío. Este complejo incluye a las subespecies cummingiana (Herbert) Bayer, hookeri, maculata Bayer y recumbens (Herbert) Bayer (Bayer 1987). Alstroemeria hookeri spp. hookeri presenta una distribución casi exclusiva en la VIII Región de Bío-Bío y se caracteriza por presentar un rizoma corto y fuerte, del cual nacen raíces almacenadoras blanquecinas, degrantamaño.Lasflores son rosadas con los tépalos superiores ornamentados de color amarillo. En esta región existen dos sectores de distribución de esta subespecie, separados por la Cordillera de la Costa, esto es, poblaciones que crecen prácticamente a orillas del mar, entre los 5 y $20 \mathrm{~m}$ de altitud en las provincias de Concepción y Ñuble y poblaciones que crecen en la vertiente oriental de la Cordillera de la Costa, entre los 100-150 m de altura, en las provincias de Bío-Bío y Ñuble. De acuerdo a lo indicado anteriormente, y por tratarse de una zona de Chile donde existen muchas especies endémicas y una alta biodiversidad vegetal, el objetivo de este trabajo fue comparar cariotípicamente poblaciones costeras y poblaciones del interior de Alstroemeria hookeri ssp. hookeri, utilizando sus cromosomas como fuente de evidencia taxonómica.

\section{MATERIALES Y METODOS}

\section{MATERIAL VEGETAL}

El material recolectado de Alstroemeria hookeri spp. hookeri proviene de 19 localidades (poblaciones) de la VIII Región del Bío-Bío de Chile (Fig. 1) y se encuentra depositado en el Herbario de la Universidad de Concepción (CONC, Tabla I). 
Análisis citogenético en Alstroemeria hookeri: CAJAS, D. ET AL.

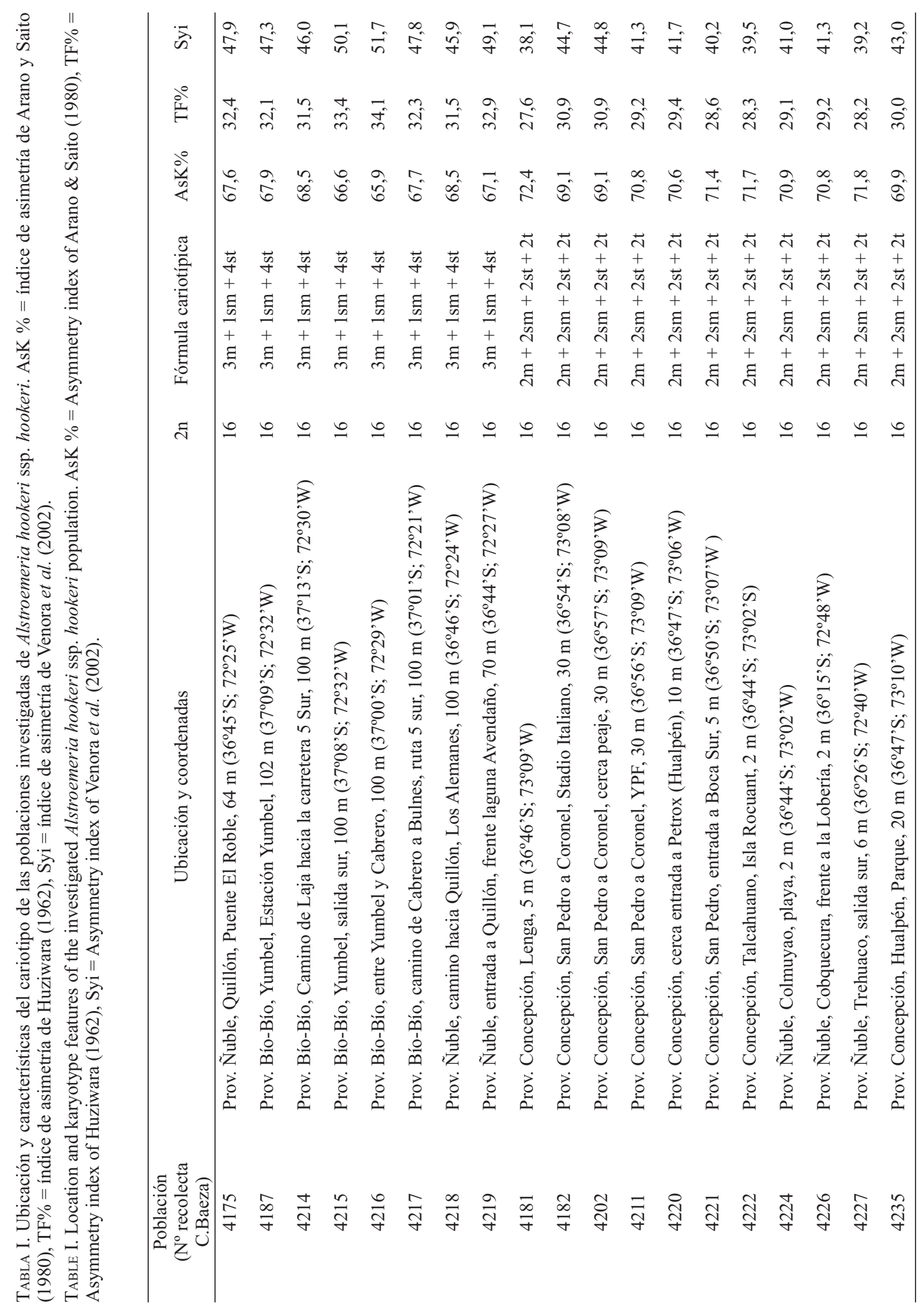




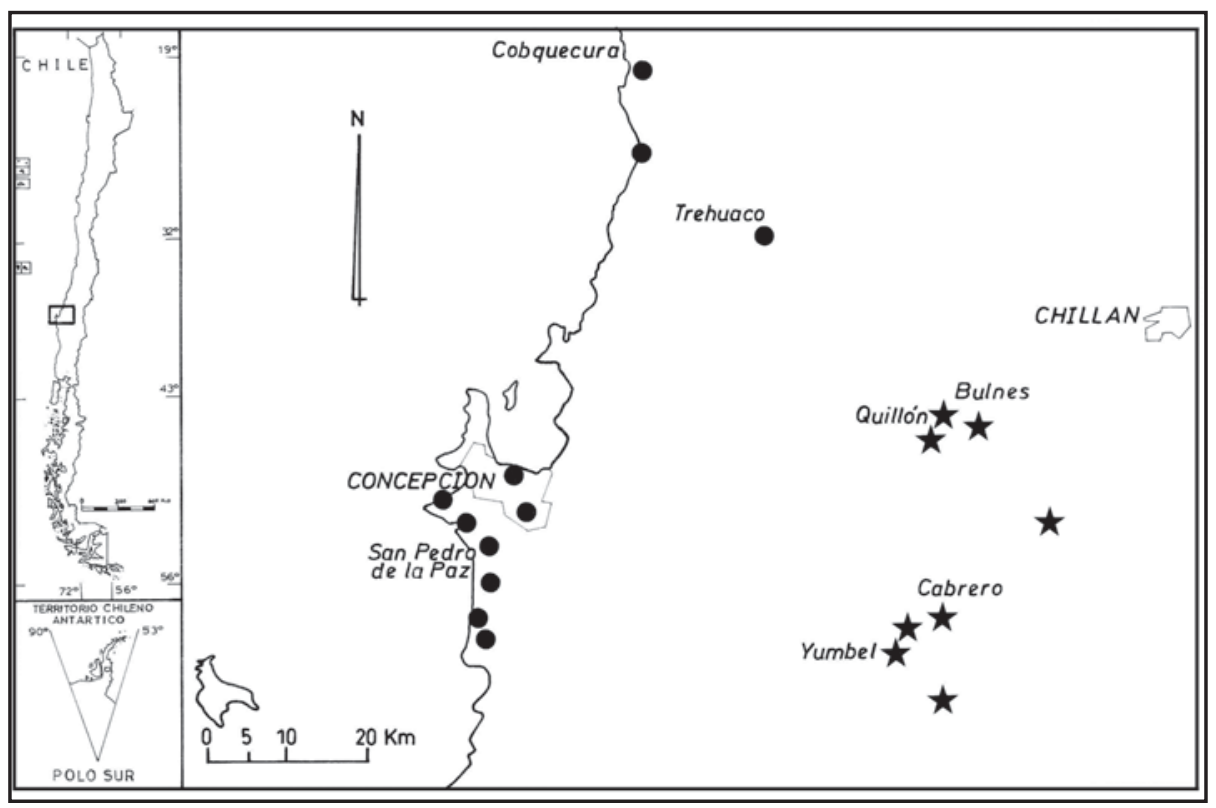

Figura 1. Ubicación geográfica de las poblaciones de Alstroemeria hookeri ssp. hookeri analizadas. Los puntos corresponden a las poblaciones de la costa; las estrellas a las poblaciones del interior.

FIGURE 1. Geographical distribution of analyzed population of Alstroemeria hookeri ssp. hookeri. Dots are coastal populations; stars are interior populations.

\section{OBTENCIÓN DE CARIOTIPOS}

Puntas de raíces de 1-2 cm de longitud, obtenidas a partir de plantas cultivadas en invernadero fueron previamente tratadas con una solución de 8-hidroxiquinoleína $(2 \mathrm{mM})$ por 24 horas a $4{ }^{\circ} \mathrm{C}$. Posteriormente, se fijaron en etanol / ácido acético (3:1) por 24 horas y se almacenaron en alcohol $70 \%$ a $-20{ }^{\circ} \mathrm{C}$. Posteriormente, para la mayoría de las poblaciones analizadas se hizo una hidrólisis ácida con $\mathrm{HCl} 0,5 \mathrm{~N}$ durante 25 minutos a $38^{\circ} \mathrm{C}$. Luego se lavó el material con agua destilada dos veces para eliminar el exceso de $\mathrm{HCl}$. Finalmente, se hizo el aplastado de la punta de la raíz en una gota de orceína acética al $1 \%$. Para las poblaciones 4181 , 4175, 4216 y 4217, las cuales fueron teñidas con DAPI posteriormente a la fijación, las raíces fueron lavadas 3 veces en agua destilada por 30 minutos, para luego ser digeridas en una mezcla de enzimas de 4\% celulasa "Onozuka R-10" (Serva) y 1\% pectiolasa Y-23 (Seishin Pharmaceutical) en $75 \mathrm{mM}$ de $\mathrm{KCl}$, a pH 4,0 por 40 minutos a $37^{\circ} \mathrm{C}$. Luego de un breve lavado en agua destilada, las raíces fueron mantenidas durante un minuto en ácido acético al $45 \%$ y luego se hizo el aplastado correspondiente.
Los cubreobjetos fueron removidos después de mantener las muestras a $-84{ }^{\circ} \mathrm{C}$. Los preparados se dejaron secar durante 1-2 días a temperatura ambiente y se almacenaron a $\quad-20 \quad{ }^{\circ} \mathrm{C}$. Los cromosomas fueron teñidos con DAPI (1,0 ng/l de 4',6-diamidino-2-fenilindol). El recuento, análisis e interpretación de los cromosomas (10 placas metafásicas) se realizó usando un microscopio Axioskop Zeiss con cámara fotográfica digital incluida. Los cromosomas se midieron con la ayuda del programa computacional "MicroMeasure 3.3" (Reeves 2001) y fueron clasificados de acuerdo a la razón de los brazos (Levan et al. 1964). Para cada población analizada, se determinó el índice de asimetría del cariotipo (AsK \%) usando la fórmula de Arano y Saito (1980), el índice de asimetría (TF\%) de Huziwara (1962) y el índice de asimetría (Syi) de Venora et al. (2002). La longitud de cada cromosoma se calculó como el porcentaje de la longitud genómica total del correspondiente juego haploide de cromosomas. Se usó el programa Corel Draw versión 8.0 para la representación gráfica de cada juego cromosómico (idiogramas), los cuales se ordenaron de acuerdo a su tamaño decreciente, 
y las fotos fueron digitalizadas o contrastadas con Corel Paint Shop Pro X. Para estudiar las relaciones de similitud entre las poblaciones analizadas se realizó un análisis de agrupamiento. Para ello, con los datos de la Tabla I se construyó una matriz de 19 poblaciones $\times 3$ caracteres, a partir de la cual se obtuvo una matriz de distancia empleando el índice de distancia taxonómica (DIST). Las poblaciones se agruparon utilizando el método de ligamiento promedio (UPGMA). Los datos fueron procesados con el programa NTSyS-PC (Numerical Taxonomic System of Multivariate Statistical Programs), diseñado por Rohlf (2000).

\section{RESULTADOS}

La Figura 1 muestra la distribución exacta de las 19 poblaciones recolectadas: corresponden al sector del interior de la VIII Región del Bío-Bío, con alturas que oscilan entre los 64 y $102 \mathrm{~m}$ y 11 corresponden al sector costero de las provincias de Concepción y Ñuble, con alturas entre los 2 y $10 \mathrm{~m}$. La Tabla I resume los resultados obtenidos en las 19 poblaciones de Alstroemeria hookeri ssp. hookeri analizadas citológicamente. Todos los individuos presentan $2 \mathrm{n}$ $=2 \mathrm{x}=16$ cromosomas. Las poblaciones del interior (C. Baeza 4175, 4187, 4214, 4215, 4216, 4217, 4218 y 4219) presentan una fórmula cariotípica haploide $3 \mathrm{~m}+1 \mathrm{sm}+4 \mathrm{st}$, es decir, tres pares de cromosomas metacéntricos, un par submetacéntrico y cuatro pares subtelocéntricos. Las poblaciones de la costa (C. Baeza 4181, 4182, 4202, 4211, 4220, 4221, 4222, 4224, 4226, 4227 у 4235) presentan una fórmula cariotípica haploide $2 \mathrm{~m}+$ $2 \mathrm{sm}+2 \mathrm{st}+2 \mathrm{t}$, es decir, dos pares de cromosomas metacéntricos, dos pares submetacéntricos, dos pares subtelocéntricos y dos pares telocéntricos (Figs. 2 y 3, Tabla I). Al comparar los promedios de los índices de asimetría entre las poblaciones costeras y las del interior se encontraron valores significativamente distintos $(\mathrm{t}=3,33$ para AsK \% y $\mathrm{t}=4,86$ para Syi con un $\mathrm{p} \leq 0,05)$. Las tablas II y III resumen el promedio de la longitud total y relativa de los cromosomas de las poblaciones costeras y del interior de $A$. hookeri ssp. hookeri. Se observa que el tamaño de los cromosomas es mucho mayor en las poblaciones del interior. Esto también se evidencia en la longitud total haploide del genoma (LTC), cuyo valor en las poblaciones del interior es de 201,2 $\pm 6,8$ y de 161,2 \pm 9,1 en las poblaciones costeras. Existe un polimorfismo interpoblacional donde la diferencia ocurre principalmente a nivel del par cromosómico 3. También existe polimorfismo en los cromosomas 4 y 8 , donde el par 4 es telocéntrico en las costeras y subtelocéntrico en las del interior y el par 8 es submetacéntrico en las costeras y subtelocéntrico en las del interior.

TaBla II. Longitud promedio de los cromosomas de A. hookeri ssp. hookeri de la costa (4181, 4182, 4202, 4211, 4220, 4221, 4224, 4226, 4227 y 4235) calculados en porcentaje de la longitud del genoma haploide total de 10 metafases para cada población. LTC haploide $(\mu \mathrm{m})=$ largo total de los cromosomas más la desviación estándar para las poblaciones costeras: $161,2 \pm 9,1$.

TABLE II. Average length of chromosomes of $A$. hookeri ssp. hookeri Coastal populations (4181, 4182, 4202, 4211, 4220, 4221, 4224, 4226, 4227 y 4235) calculated in percent of the mean haploid genome length of 10 metaphases for each population. TCL haploid $(\mu \mathrm{m})=$ total chromosom lenght plus standar deviation for Coastal population: 161.2 \pm 9.1 .

\begin{tabular}{ccccccc}
\hline Par cromosómico & $\begin{array}{c}\text { Brazo largo } \\
(\%) \pm \text { D.E. }\end{array}$ & $\begin{array}{c}\text { Brazo corto } \\
(\%) \pm \text { D.E. }\end{array}$ & $\begin{array}{c}\text { Largo relativo } \\
(\%)\end{array}$ & $\begin{array}{c}\text { Largo total } \\
(\mu \mathrm{m})\end{array}$ & $\begin{array}{c}\text { Radio del brazo } \\
(\mathrm{L} / \mathrm{C})\end{array}$ & $\begin{array}{c}\text { Tipo de } \\
\text { cromosoma }\end{array}$ \\
\hline 1 & $5,74 \pm 0,26$ & $5,42 \pm 0,20$ & 11,16 & 20,16 & 1,06 & $\mathrm{~m}$ \\
2 & $4,27 \pm 0,14$ & $2,96 \pm 0,10$ & 7,23 & 13,06 & 1,44 & $\mathrm{~m}$ \\
3 & $5,57 \pm 0,28$ & $0,63 \pm 0,10$ & 6,20 & 11,20 & 8,84 & $\mathrm{t}$ \\
4 & $5,16 \pm 0,22$ & $0,68 \pm 0,10$ & 5,84 & 10,55 & 7,59 & $\mathrm{t}$-sat \\
5 & $4,71 \pm 0,10$ & $0,77 \pm 0,00$ & 5,48 & 9,90 & 6,12 & $\mathrm{st}$ \\
6 & $4,38 \pm 0,32$ & $0,90 \pm 0,20$ & 5,28 & 9,54 & 4,87 & st-sat \\
7 & $3,09 \pm 0,26$ & $1,45 \pm 0,10$ & 4,54 & 8,20 & 2,13 & sm \\
8 & $2,87 \pm 0,10$ & $1,45 \pm 0,10$ & 4,32 & 7,80 & 1,98 & $\mathrm{sm}$ \\
\hline
\end{tabular}



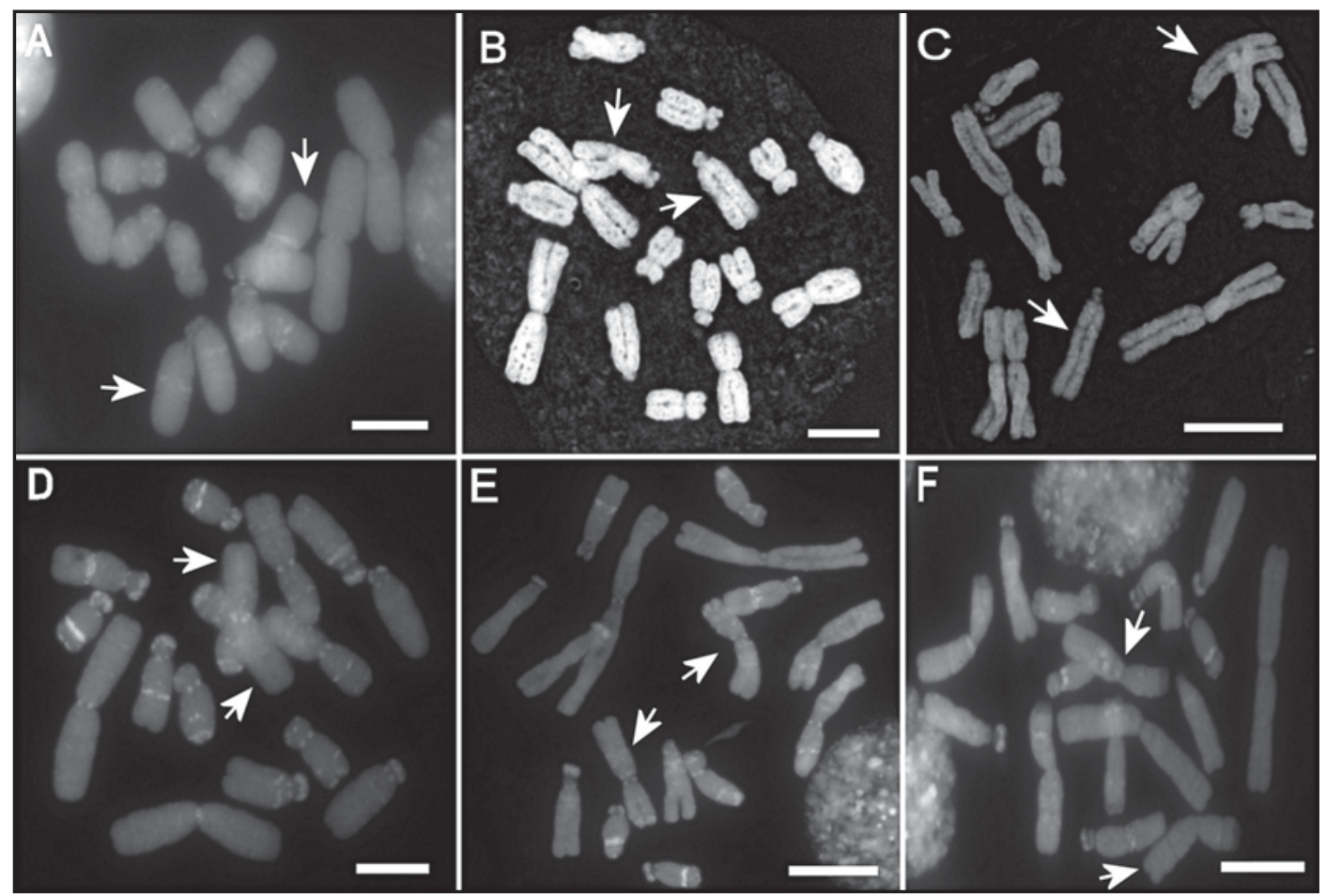

FIgURA 2. Placas metafásicas de las poblaciones de Alstroemeria hookeri ssp. hookeri analizadas $(2 \mathrm{n}=16)$. $\mathrm{A}=\mathrm{Baeza}$ 4181. $\mathrm{B}=$ Baeza 4211. $\mathrm{C}=$ Baeza 4224 (Poblaciones costeras). $\mathrm{D}=$ Baeza 4175. $\mathrm{E}=$ Baeza 4216. $\mathrm{F}=\mathrm{Baeza} 4217$ (Poblaciones del interior). Las flechas indican el cromosoma 3. Escala: $10 \mu \mathrm{m}$ (A, D, E, F tinción con DAPI, B y C tinción con orceína acética).

FIGURE 2. Metaphasic chromosomes of Alstroemeria hookeri ssp. hookeri analized populations $(2 \mathrm{n}=16)$. $\mathrm{A}=\mathrm{Baeza}$ 4181. $\mathrm{B}=$ Baeza 4211. $\mathrm{C}=$ Baeza 4224 (Coastal populations). $\mathrm{D}=$ Baeza 4175. $\mathrm{E}=$ Baeza 4216. $\mathrm{F}=$ Baeza 4217 (Interior populations). Arrows are indicating the chromosome 3. Scale $=10 \mu \mathrm{m}$ (A, D, E, F with DAPI, B and C with orcein).

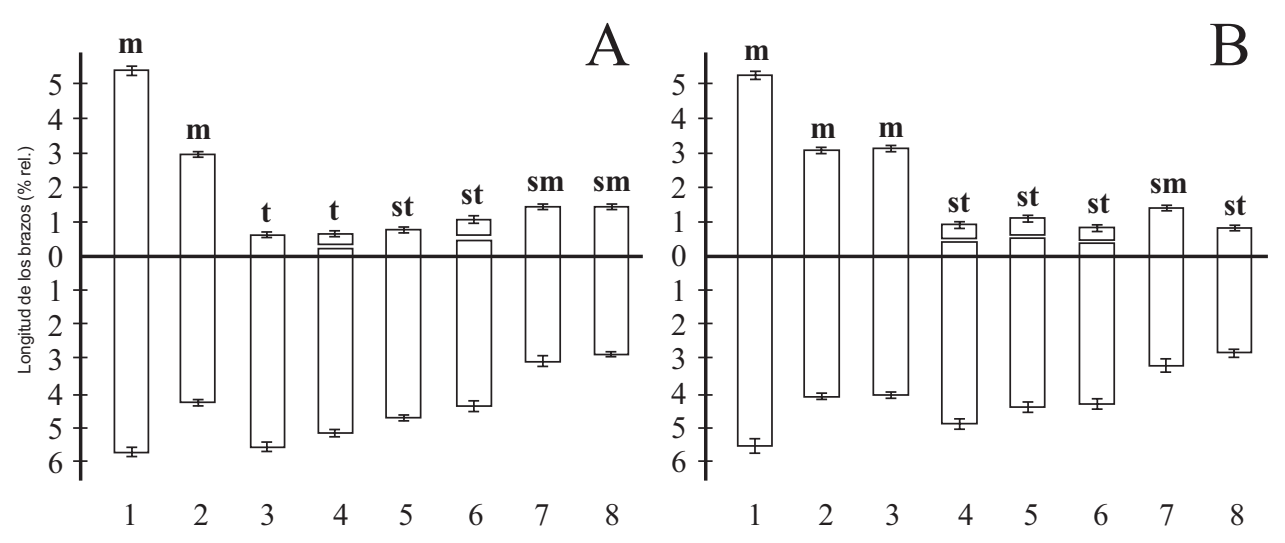

FIGURA 3. Idiogramas haploides de Alstroemeria hookeri ssp. hookeri. A= poblaciones costeras. $\mathrm{B}=$ poblaciones del interior. Los cromosomas se han ordenado de acuerdo a su tamaño decreciente.

Figure 3. Ideograms of the haploid chromosomes complement of Alstroemeria hookeri ssp. hookeri. A= Coastal populations. $\mathrm{B}=$ Interior populations. The chromosomes have been ordered according to decreasing size. 
Las relaciones de similitud entre todas las poblaciones analizadas se representan en el fenograma de la figura 4, donde se puede apreciar la separación de todas las poblaciones en dos grupos. Uno de ellos (A) reúne a todas las poblaciones distribuidas en poblaciones del interior y el otro grupo (B) a todas las poblaciones costeras. El índice de correlación cofenética (r) resultó ser 0,87 , lo que indica que existe poca distorsión de los datos de similitud en la representación del fenograma.

TABLA III. Longitud promedio de los cromosomas de A. hookeri ssp. hookeri de poblaciones del interior $(4175,4187$, 4214, 4215, 4216, 4217, 4218 y 4219) calculados en porcentaje de la longitud del genoma haploide total de 10 metafases para cada población. LTC haploide $(\mu \mathrm{m})=$ largo total de los cromosomas más la desviación estándar para las poblaciones del interior: $201,2 \pm 6,8$.

TABLE III. Average length of chromosomes of $A$. hookeri ssp. hookeri Interior populations $(4175,4187,4214,4215,4216$, 4217,4218 y 4219) calculated in percent of the mean haploid genome length of 10 metaphases for each population. TCL haploid $(\mu \mathrm{m})=$ total chromosome lenght plus standar deviation for Interior population: 201.2 \pm 6.8 .

\begin{tabular}{ccccccc}
\hline $\begin{array}{c}\text { Par } \\
\text { cromosómico }\end{array}$ & $\begin{array}{c}\text { Brazo largo } \\
(\%) \pm \text { D.E. }\end{array}$ & $\begin{array}{c}\text { Brazo corto } \\
(\%) \pm \text { D.E. }\end{array}$ & $\begin{array}{c}\text { Largo relativo } \\
(\%)\end{array}$ & $\begin{array}{c}\text { Largo total } \\
(\mu \mathrm{m})\end{array}$ & $\begin{array}{c}\text { Radio del brazo } \\
(\mathrm{L} / \mathrm{S})\end{array}$ & $\begin{array}{c}\text { Tipo de } \\
\text { cromosoma }\end{array}$ \\
\hline 1 & $5,56 \pm 0,42$ & $5,23 \pm 0,22$ & 10,79 & 27,05 & 1,06 & $\mathrm{~m}$ \\
2 & $4,14 \pm 0,20$ & $3,07 \pm 0,10$ & 7,21 & 18,08 & 1,35 & $\mathrm{~m}$ \\
3 & $4,08 \pm 0,14$ & $3,12 \pm 0,14$ & 7,20 & 18,05 & 1,31 & $\mathrm{~m}$ \\
4 & $4,93 \pm 0,26$ & $0,84 \pm 0,17$ & 5,77 & 14,47 & 5,87 & st-sat \\
5 & $4,45 \pm 0,28$ & $1,17 \pm 0,14$ & 5,62 & 14,09 & 3,80 & st-sat \\
6 & $4,33 \pm 0,28$ & $0,90 \pm 0,14$ & 5,23 & 13,11 & 4,81 & st-sat \\
7 & $3,23 \pm 0,39$ & $1,39 \pm 0,10$ & 4,62 & 11,58 & 2,32 & sm \\
8 & $2,85 \pm 0,22$ & $0,80 \pm 0,14$ & 3,65 & 9,15 & 3,56 & st \\
\hline
\end{tabular}

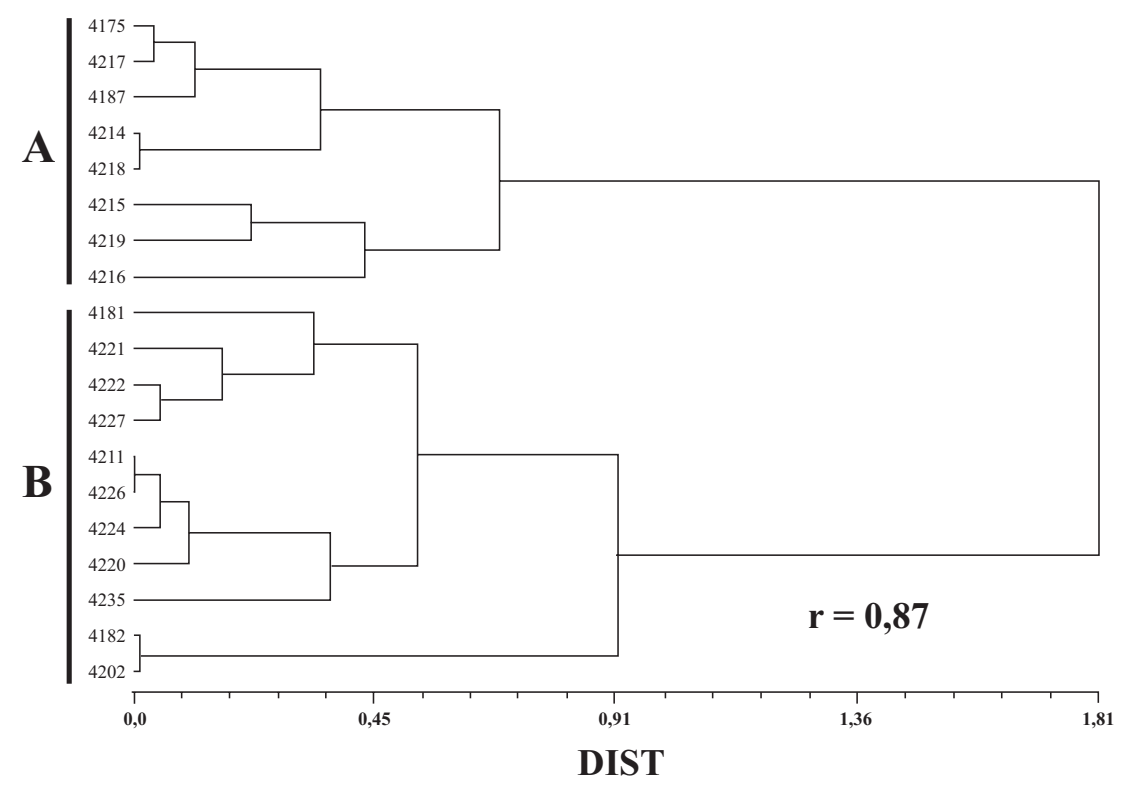

FIgURA 4. Fenograma generado vía DIST y ligamiento UPGMA para las poblaciones de Alstroemeria hookeri ssp. hookeri analizadas. A: poblaciones del interior, B: poblaciones costeras.

FIGURE 4. Phenogram using UPGMA and DIST for the Alstroemeria hookeri ssp. hookeri analized populations. A: Interior populations, B: coastal populations. 


\section{DISCUSION}

Alstroemeria hookeri ssp. hookeri crece en suelos arenosos con bajo contenido orgánico, pero de buen drenaje. Forma poblaciones con un gran número de individuos y su período de floración ocurre entre diciembre y enero (Muñoz \& Moreira 2003). Esta subespecie se distribuye en un área reconocida como uno de los 25 hotspots mundiales de biodiversidad (Myers et al. 2000).

El análisis citogenético comparado efectuado entre las poblaciones costeras y las del interior evidenció la presencia de dos citotipos diferentes. Las poblaciones costeras presentaron siempre cromosomas con una fórmula cariotípica formada por dos pares metacéntricos (los mayores del complemento cromosómico), dos pares submetacéntricos, dos pares subtelocéntricos y dos pares telocéntricos (Figs. 2 y 3 ), en cambio las poblaciones del interior siempre presentaron una fórmula cariotípica formada por tres pares metacéntricos (los mayores del complemento cromosómico), un par submetacéntrico y cuatro pares subtelocéntricos. Las poblaciones costeras presentan un cariotipo más asimétrico que las poblaciones del interior, con valores de $\mathrm{AsK} \%$ que fluctúan entre 69,1 a 72,5, en cambio en las poblaciones del interior estos valores van desde 65,9 a 68,5 (Tabla I). Se observa un marcado polimorfismo interpoblacional a nivel del cromosoma 3 entre las poblaciones costeras y del interior. El polimorfismo es una condición que también se da en otras plantas vasculares, como es el caso de Brachycome Cass. (Asteraceae) (Houben et al. 2000), Scilla L. (Liliaceae) (Greilhuber \& Speta 1976) y Placea amoena Phil. (Amaryllidaceae) (Baeza \& Schrader 2004), entre otras.

Nuestras observaciones indican que el cariotipo en $A$. hookeri ssp. hookeri no es uniforme entre poblaciones y que cambios a nivel cromosomal han contribuido a la diversificación de esta subespecie en el área de estudio, situación similar también encontrada en Crotalaria L. (Fabaceae) donde se encontró que en poblaciones con el mismo nivel de ploidía hubo diferencias cuantitativas en los parametros morfológicos del cariotipo (Almada et al. 2006). Si comparamos los índices de asimetría del cariotipo (AsK\%, TF\% y Syi), observamos que las diferencias entre ellos quedan de manifiesto al correlacionar las poblaciones estudiadas y su distribución geográfica, de tal manera que el análisis de agrupamiento permite evidenciar dos grandes grupos, que se corresponden con los citotipos antes señalados (Fig. 4). Los rearreglos estructurales de los cromosomas mantienen el número cromosómico constante, pero las variaciones pueden ocurrir entre los cariotipos dentro de un género o de especies (Schubert \& Rieger 1985). Esta situación ha sido observada en Boronia Sm. (Rutaceae), cuya variación en la asimetría de sus cariotipos estaría indicando translocación de segmentos cromosómicos y las diferencias en el LTC entre poblaciones de la misma especie con el mismo número cromosómico podrían indicar duplicación o deleción de segmentos (Shan et al. 2003). El cromosoma 3 conserva su longitud relativa en ambos cariotipos y sólo cambia la posición del centrómero (y la forma cromosómica), lo que sugiere más bien una inversión pericéntrica. Al comparar los índices de asimetría observados y el tamaño total del genoma haploide, se observa que en las poblaciones costeras (Tabla II) este valor fue de $161,2 \pm 9,1$ y de 201,2 $\pm 6,8$ en las poblaciones del interior (Tabla III). Estos valores sugieren que durante el proceso de divergencia entre las poblaciones costeras y del interior hubo un cambio en el LTC de las poblaciones. Por lo tanto, la variación del cariotipo en $A$. hookeri ssp. hookeri es importante no sólo como fuente de variabilidad genética sino también porque ella representa un importante rasgo micromorfológico para comprender la evolución del cariotipo en esta subespecie que ocupa un área considerada como un hotspot dentro de la flora chilena. Variaciones similares del cariotipo han sido reportadas en especies del genero Koelreuteria (Sapindaceae) donde se observó una diferenciación en el cariotipo en dos de sus especies (Urdampilleta et al. 2005).

Es probable que el aislamiento geográfico entre las poblaciones comparadas, producto de la presencia de la Cordillera de la Costa, haya permitido la diferenciación poblacional entre las poblaciones del rango costero y las del interior en la Región del Bío-Bío en Chile. Estas diferencias citogenéticas entre las poblaciones estudiadas es coincidente con la estructuración poblacional obtenida a través del uso de isoenzimas y de marcadores RAPD (Ruiz et al., datos no publicados) como también del análisis morfológico detallado de las flores y de caracteres vegetativos (Negritto et al., datos no publicados). Todos estos resultados estarían evidenciando la existencia de una nueva subespecie dentro del complejo A. hookeri en Chile, situación de mucho interés por el enorme potencial ornamental que presenta estos taxa. 


\section{AGRADECIMIENTOS}

Trabajo financiado por Fondecyt 1070520. También se agradece al Departamento de Botánica de la Universidad de Concepción por todas las facilidades otorgadas.

\section{BIBLIOGRAFIA}

Aagesen, L. \& M. SAnso. 2003. The phylogeny of the Alstroemeriaceae, based on morphology, rps16 Intron, and rbcL sequence data. Systematic Botany 28(1): 47-69.

AKER, S. \& W. Healy. 1990. The phytogeography of the genus Alstroemeria. Herbertia 46: 76-87.

Almada, R., J. Daviña \& J. Seijo. 2006. Karyotype analysis and chromosome evolution in southernmost South American species of Crotalaria (Leguminosae). Botanical Journal of the Linnean Society 150(3): 329-342.

Arano, H. \& H. Saito. 1980. Cytological studies in family Umbelliferae 5. Karyotypes of seven species in subtribe Seselinae. La Kromosomo 2(17): 471-480.

Arroyo, M.T.K. 1995. Plantas, hongos y líquenes. En: J. Simonetti, M.T.K. Arroyo, A. Spotorno \& E. Lozada (eds.), Diversidad biológica de Chile, 57 pp. Conicyt, Santiago, Chile.

Baeza, C.M. \& O. Schrader. 2004. Karyotype analysis of Placea amoena Phil. (Amaryllidaceae) by double fluorescence in situ hybridization. Caryologia 57(2): 209-214.

Baeza, C.M., O. Schrader, E. Ruiz \& M. Negritto. 2006. Análisis comparativo del cariotipo en poblaciones de Alstroemeria ligtu subsp. ligtu y $A$. ligtu subsp. simsii (Alstroemeriaceae) de Chile. Darwiniana 44(2): 313-318.

Baeza, C.M., O. Schrader \& H. Budahn. 2007a. Characterization of geographically isolated accessions in five Alstroemeria L. species (Chile) using FISH of tandemly repeated DNA sequences and RAPD analysis. Plant Systematics and Evolution 269: 1-14.

Baeza, C.M., O. Schrader, E. Ruiz \& M. Negritto. 2007b. Análisis comparativo del cariotipo en poblaciones de Alstroemeria aurea R. Graham (Alstroemeriaceae) de Chile. Gayana Botánica 64(1): 31-37.

BAYER, E. 1987. Die Gattung Alstroemeria in Chile. Mitteilungen der Botanischen Staatssammlung München 24: 1-362.

BuitendiJK, J. \& M. Ramanna. 1996. Giemsa C-banded karyotypes of eight species of Alstroemeria L. and some of their hybrids. Annals of Botany 78: 449-457.

Buitendijk, J., E. Boon \& M. Ramanna. 1997. Nuclear DNA content in twelve species of Alstroemeria L. and some of their hybrids.
Annals of Botany 79: 343-353.

Buitendijk, J., A. Peters, R. Jan-Quené \& M. Ramanna. 1998. Genome size variation and C-band polymorphism in Alstroemeria aurea, A. ligtu and $A$. magnifica (Alstroemeriaceae).

Plant Systematics and Evolution 212: 87-106.

De Jeu, M., J. Lasschuit, A. Kuipers, S. Kamstra \& R. VISSER. 1997. Characterisation and localization of repetitive DNA sequences in the ornamental Alstroemeria aurea Graham. Theoretical and Applied Genetic 94: 982-990.

Greilhuber, J. \& F. Speta. 1976. C-banded karyotypes in the Scilla hohenackeri group S. persica, and Poschkina (Liliaceae). Plant Systematics and Evolution 126:149-188.

Houben, A., G. Wanner, L. Hanson, D. Verlin, C. Leach. $\&$ J. TIMMES. 2000. Cloning and characterization of polymorphic heterochromatic segments of Brachycome dichromosomatica. Chromosoma 109: 206-213.

Huziwara, Y. 1962. Karyotype analysis in some genera of Compositae. VIII. Further studies on the chromosomes of Aster. American Journal of Botany 49: 116-119.

Jara, P., C. Palma \& E. Von Brand. 2004. Karyotype and C-bands in the annual inca lily Alstroemeria graminea. Belgien Journal of Botany 137(2): 199-204.

Kamstra, S., A. Kuipers, M. De Jeu, M. Ramanna \& E. JACOBSEN. 1997. Physical localisation of repetitive DNA sequences in Alstroemeria: karyotyping of two species with species-specific and ribosomal DNA. Genome 40: 652-658.

Kuipers, A., D. Van Os, H. De Jong \& M. Ramanna. 1997. Molecular cytogenetics of Alstroemeria: identification of parental genomes in interspecific hybrids and characterization of repetitive DNA families in constitutive heterochromatin. Cromosome Research 5: 31-39.

Kuipers, A., J. Heslop-Harrison \& E. Jacobsen. 1998. Characterisation and physical localisation of Ty1copia-like retrotransposons in four Alstroemeria species. Genome 41: 357-367.

Kuipers, A., S. Kamstra, M. De Jeu \& R. Visser. 2002. Molecular characterisation and physical localization of highly repetitive DNA sequences from Brazilian Alstroemeria species. Chromosome Research. 389-398.

LeVAn, A., K. Fredga \& A. SANDBERg. 1964. Nomenclature for centromeric position on chromosomes. Hereditas 52: 201-220.

Muñoz, M. \& A. MoreIra. 2003. Alstroemerias de Chile. Diversidad, distribución y conservación. Taller La Era, Santiago. 140 pp.

Myers, N., R. Mittermeier, C. Mittermeier, G. Fonseca \& J. Kent. 2000. Biodiversity hotspots for conservation priorities. Nature 403: 853-858.

Ravenna, P. 1988. New or noteworthy species of Alstroemeria. Phytologia 64: 281-288.

Reeves, A. 2001. MicroMeasure: a new computer program for the collection and analysis of 
Gayana Bot. 66(2), 2009

cytogenetic data. Genome 44: 239-443.

RoHLF, F. 2000. NTSYS-PC Numerical taxonomy and multivariate analysis system. Version 2.1. Manual. Applied Biostatistics, New York, USA.

Sanso, A. 2002. Chromosome studies in Andean taxa of Alstroemeria (Alstroemeriaceae). Botanical Journal of the Linnean Society 138: 451-459.

Sanso, A. \& J. HunziKer. 1998. Karyological studies in Alstroemeria and Bomarea (Alstroemeriaceae). Hereditas 129: 67-74.

Schubert, I. \& R. Rieger. 1985. A new mechanism for altering chromosome number during karyotype evolution. Theoretical and Applied Genetic 70: 213-221.

Shan, F., G. Yan \& J. Plummer. 2003. Karyotype evolution in the genus Boronia (Rutaceae). Botanical Journal of the Linnean Society 142(3): 309-320.

Stephens, J., T. Tsuchiya \& H. Hughes. 1993. Chromosome studies in Alstroemeria pelegrina L. International Journal of Plant Science 154(4): 565-571.

Strasburger, E. 1882. Über den Teilungsvorgang der
Zellkerne und das Vehaltnis der Kernteilung zur Zellteilung. Archiv für Mikrobiologie und Anatomie 21: 476-596.

Tsuchiya, T. \& A. Hang. 1989. Cytogenetics in the genus Alstroemeria. Herbertia 45: 163-170.

Urdampilleta, J., M. Ferrucci \& A. Vanzela. 2005. Karyotype differentiation between Koelreuteria bipinnata and K. elegans subsp. formosana (Sapindaceae) based on chromosome banding patterns. Botanical Journal of the Linnean Society 149(4): 451-455.

Venora, G., S. Blangiforti, M. Ruffini Castiglioni, D. Pignone, F. Losavio \& R. Cremonini. 2002. Chromatin organisation and computer arded karyotyping of Triticum durum Desf. cv Timilia. Caryologia 55: 91-98.

Zhou, S., M. De Jeu, R. Visser \& A. Kuipers. 2003. Characterisation of distant Alstroemeria hybrids: application of highly repetitive DNA sequences from A. ligtu subsp. ligtu. Annals of Applied Biology 142(3): 277-283.

Recibido: 05.03.09

Aceptado: 04.05.09 